\title{
Peptide formation as on the early Earth: from amino acid mixtures to peptides in sulphur dioxide
}

\section{Oliver Trapp ( $\nabla$ oliver.trapp@cup.uni-muenchen.de )}

Ludwig Maximilian University of Munich https://orcid.org/0000-0002-3594-5181

\section{Fabian Sauer}

Ludwig Maximilian University of Munich

\section{Maren Haas}

Ludwig Maximilian University of Munich

\section{Constanze Sydow}

Ludwig Maximilian University of Munich

\section{Alexander Siegle}

Ludwig Maximilian University of Munich

\section{Christoph Lauer}

Ludwig Maximilian University of Munich

\section{Article}

Keywords: Peptide Bonds, Active Polymers Development, Salt-induced Peptide Formation, Abiotic Peptide Synthesis, Covellite

Posted Date: July 12th, 2021

DOI: https://doi.org/10.21203/rs.3.rs-629724/v1

License: (c) (1) This work is licensed under a Creative Commons Attribution 4.0 International License.

Read Full License

Version of Record: A version of this preprint was published at Nature Communications on December 10th, 2021. See the published version at https://doi.org/10.1038/s41467-021-27527-7. 


\section{Peptide formation as on the early Earth: 2 from amino acid mixtures to peptides in 3 sulphur dioxide 4}

5 Fabian Sauer ${ }^{1}$, Maren Haas $^{1,2}$, Constanze Sydow ${ }^{1}$, Alexander F. Siegle ${ }^{1}$, Christoph A. Lauer ${ }^{1}$, Oliver $6 \operatorname{Trapp}^{* 1,2}$

${ }^{1}$ Department of Chemistry and Pharmacy, Ludwig-Maximilians-University, Butenandtstr. 5-13, 81377

Munich, Germany

${ }^{2}$ Max-Planck-Institute for Astronomy, Königstuhl 17, 69117 Heidelberg, Germany

*Corresponding author: oliver.trapp@cup.uni-muenchen.de

Fabian Sauer: fabian.sauer@cup.uni-muenchen.de; https://orcid.org/0000-0002-0869-6837

Dr. Maren Haas: maren.haas@cup.uni-muenchen.de; https://orcid.org/0000-0002-4712-7338

Constanze Sydow: constanze.sydow@cup.uni-muenchen.de; https://orcid.org/0000-0003-4947-4103

Dr. Alexander F. Siegle: alexander.siegle@cup.uni-muenchen.de; https://orcid.org/0000-0003-0955-

Christoph A. Lauer: ch.lauer@campus.Imu.de; $\underline{\text { https://orcid.org/0000-0002-2708-9610 }}$ 


\section{1 1 Abstract}

The formation of peptide bonds is one of the most important biochemical reaction steps. Without the development of structurally and catalytically active polymers, there would be no life on our planet. Intensive research is being conducted on possible reaction pathways for the formation of complex peptides on the early Earth. Salt-induced peptide formation (SIPF) by metal catalysis is one possible pathway for abiotic peptide synthesis. The high salt concentration supports dehydration in this process. However, the formation of large, complex oligomer systems is prevented by the high thermodynamic barrier of peptide condensation in aqueous solution. Liquid sulphur dioxide proves to be a superior alternative for copper-catalysed peptide condensation. Compared to water, the amino acids are activated in sulphur dioxide, which leads to the incorporation of all 20 proteinogenic amino acids into the resulting proteins and thus to a large variety of products. Strikingly, even extremely low initial reactant concentrations of only $50 \mathrm{mM}$ are sufficient for extensive peptide formation, leading to an overall yield of $2.9 \%$ for dialanine in 7 days. The reactions carried out at room temperature and the successful use of the Hadean mineral covellite as a catalyst, suggest a volcanic environment for the formation of the peptide world on early Earth as a likely scenario. 


\section{Introduction}

Because of the great variety of functions peptides can perform in nature, the formation of peptides from amino acids is irreplaceable for life as we know it ${ }^{1}$. This reaction plays a key role in the cellular function as well as in the origin of life. Several likely scenarios for prebiotic amino acid syntheses have been proposed $d^{2-5}$. In addition, amino acids have been identified on meteorites ${ }^{6-9}$. The original peptide synthesis from prebiotic material is being intensively researched today and numerous possible synthetic pathways have been proposed. The formation of peptides from amino acids is not readily performed in aqueous solution because condensation reactions are thermodynamically unfavourable under these conditions. Proposed scenarios to overcome this barrier include drying of amino acid mixtures $^{10,11}$, mineral surface catalysis ${ }^{12,13}$, co-condensation with $\alpha$-hydroxy acids ${ }^{14}$, polymerisation of activated amino acid derivatives ${ }^{15,16}$ and solvent-free mechanochemical environments ${ }^{17}$. In salt induced peptide formation (SIPF), highly concentrated saline solutions provide dehydrating conditions, while additional metal ions, preferably copper(II), further enhance the kinetics of peptide formation 18,19. The availability of copper(II) depends on the oxidation state of the Hadean atmosphere and would only have been possible at oxygen partial pressures of $10^{-35} \mathrm{~atm}^{20}$. Several copper minerals are assumed to have been present on the early Earth, particularly sulphides such as covellite ${ }^{21}$.

To circumvent the thermodynamic barrier in aqueous solution, other solvents were also considered for prebiotic peptide formation. Some alternatives such as volcanic, magmatic conditions ${ }^{22}$, formamide as solvent $\mathrm{t}^{23,24}$ or eutectic solutions have been examined in the past ${ }^{25,26}$. Moreover, sulphur dioxide $\left(\mathrm{SO}_{2}\right)$ has gained attention as a prebiotically available compound in recent years, as it has been released in significant amounts into the atmosphere by volcanic outgassing and is also present in large quantities in other celestial bodies ${ }^{27-29}$. Local concentration maxima, the low boiling point as well as the low vapour pressure suggest the possibility of liquid $\mathrm{SO}_{2}$ ponds near volcanoes on the Hadean Earth $^{30,31}$, especially since it would probably have had a higher total atmospheric pressure ${ }^{32}$. The influence of $\mathrm{SO}_{2}$ has been studied in various prebiotic reactions, but mostly sulphite salts in water were used instead of liquid $\mathrm{SO}_{2}{ }^{33-35}$. 
Systems of higher complexity are of particular interest in the formation of peptides, as they allow the preferential formation of specific peptide sequences, functional polymers and even their further enrichment ${ }^{36}$. Prebiotic amino acid syntheses also typically lead to a variety of products ${ }^{3}$, whose conversion results in a high diversity of peptides, depending on the reaction conditions. In recent studies, however, often only a limited number of amino acids are used simultaneously in the syntheses. The use of a larger number of amino acids in the same reaction reveals many additional aspects of the synthesis method under investigation, including frequent by-products or lack of compatibility with the reaction conditions or the other amino acids. On the other hand, it also reveals the selectivity to form certain peptides whose sequence may have greater potential for functionality due to the greater likelihood of formation.

In the present work, we investigate condensed $\mathrm{SO}_{2}$ as an alternative hygroscopic solvent compared to aqueous salt solutions for prebiotic peptide formation. Complex mixtures of amino acids are reacted in both systems and the resulting product distributions are compared. It is shown that the different environments produce varying selectivity in the peptide sequences. However, peptide condensation in $\mathrm{SO}_{2}$ proceeds under simpler conditions, at room temperature and with low amino acid and metal catalyst loadings. Di- to tetrapeptides are formed and, importantly, all 20 amino acids can be incorporated.

\section{Results}

\subsection{Peptide formation in sulphur dioxide}

Although the advantages of pure liquid $\mathrm{SO}_{2}$ as a solvent in organic synthesis are well known ${ }^{37}$, its use in the search for peptide formation reactions under prebiotic conditions has not yet been considered. Starting with the simplest amino acids glycine $(G)$ and L-alanine $(A)$, we investigated the possibility of efficient peptide condensation in liquid $\mathrm{SO}_{2}$ by metal catalysis. For this purpose, the two amino acids were mixed with $\mathrm{CuCl}_{2}$ in a pressure apparatus, $\mathrm{SO}_{2}$ was added, and the mixture was stirred for 1-21 d 
at room temperature. The reaction mixture was analysed by capillary electrophoresis (CE) and highpressure liquid chromatography (HPLC), both coupled with high resolution mass spectrometry (HRMS). No peptides were observed without $\mathrm{CuCl}_{2}$ as additive. When $\mathrm{CuCl}_{2}$ was added, glycylglycine (GG) was already formed after $1 \mathrm{~d}$; alanine-containing dipeptides as well as traces of the tripeptide GGG were detected after $7 \mathrm{~d}$. Longer reaction times led to a further increase in peptide products. The cyclic diketopiperazines, which are often observed as a by-product in peptide condensation reactions ${ }^{38}$, were not detected.

The next step was to investigate the effect of additives to the reaction mixture. High amounts of $\mathrm{NaCl}$ enable the SIPF reaction in water and were therefore also investigated in this context. $\mathrm{NaCl}$ did not increase the reactivity, on the contrary it inhibited the product formation at high salt concentrations, similar to the reaction in water.

Another additive that was investigated is urea. Its prebiotic synthesis has already been described ${ }^{39,40}$. Urea has already been used as a condensation agent in prebiotic phosphorylation reactions with nucleosides ${ }^{41,42}$. The exact mechanism of the enhancing effect is not clear, but the hydrolysis of urea and the formation of reactive intermediate species are under discussion ${ }^{41}$. In addition, urea is known to form strong hydrogen bonds, denature proteins and remove water from the hydration shell of proteins $^{43}$. A positive effect of urea on peptide condensation through the formation of reactive intermediates or as a dehydrating agent is therefore conceivable. In fact, the addition of urea to peptide formation in $\mathrm{SO}_{2}$ not only led to an enhancement of already detected peptide signals, but it also supported the formation of the previously undetected tripeptides AAA, AAG and AGG. The use of urea without a metal catalyst did not lead to peptide formation. Thus, urea does not catalyse the reaction itself, but promotes it as a condensation agent. For the further investigations, an equivalent of urea was added to all reactions. The peptide yields as a function of reaction time are shown in Fig. 1a. Significant amounts of dipeptides were already formed after $1 \mathrm{~d}$ and, except for $\mathrm{GG}$, the yields increased from then until $21 \mathrm{~d}$. The concentration of GG remained rather constant after $3 \mathrm{~d}$, but 
significant amounts of tripeptides also appeared after $7 \mathrm{~d}$. It is possible that GG is converted to tripeptides more rapidly than the other dipeptides.

A critical point in prebiotic reactions is often the required high concentration of reactants, which cannot be reconciled with a dilute ocean or pond on early Earth. We started the reaction with a typical concentration of $400 \mathrm{mM}$ per amino acid and $\mathrm{CuCl}_{2}$, but also tested lower concentrations of $200 \mathrm{mM}$, $100 \mathrm{mM}$, and $50 \mathrm{mM}$. Contrary to our expectations, the reaction was favoured at lower concentrations, leading to the formation of more tri- and even tetrapeptides at $50 \mathrm{mM}$ after $21 \mathrm{~d}$. Fig. $1 \mathrm{~b}$ shows the dipeptide yields after $7 \mathrm{~d}$ as a function of the initial amino acid and $\mathrm{CuCl}_{2}$ concentrations. With decreasing amino acid concentration, the dipeptide yields remained constant. The maximum yield for AA was observed at an initial reactant concentration of $50 \mathrm{mM}$, suggesting effective peptide synthesis even in dilute solutions. Furthermore, significant amounts of tri- and tetrapeptides could be detected after $21 \mathrm{~d}$. To further investigate the reaction in dilute solutions, we also reduced the catalyst loading. If the metal salt was not used stoichiometrically, but in $1 \mathrm{~mol} \%$, the reaction still led to dipeptide formation.

To further test the plausibility of the reaction under prebiotic conditions, we replaced the synthetic $\mathrm{CuCl}_{2}$ catalyst with a natural sample of the Hadean copper(II) mineral covellite (CuS). With mineral catalysis in the presence of urea, dipeptides GG, AA, GA and AG were formed from $400 \mathrm{mM} \mathrm{A}$ and $\mathrm{G}$ after $7 \mathrm{~d}$ (Supplementary Fig. 19). Tripeptides were not detected. Covellite thus catalyses the reaction despite a somewhat slower turnover, supporting the importance of this reaction scenario for the early Earth.

\subsection{Application to complex amino acid mixtures}

Prebiotic peptide formation is often tested for only a limited number of amino acids. If many compounds are used, however, they are usually tested individually in separate reactions rather than in a mixture. However, these concepts overlook the dynamics and possible interactions of all amino 
acids with each other, which must inevitably also have taken place under the complex conditions of the early Earth. Therefore, the study of complex larger systems is becoming increasingly important. This approach allows the whole spectrum of products to be studied under specific reaction conditions and compared with others. The development of more sophisticated analytical tools allows the detailed study of the immense number of products generated during peptide condensation (peptide coupling of all proteinogenic amino acids with each other already yields 400 possible dipeptide and 8000 tripeptide sequences). The combination of efficient CE separations with fast, high-resolution mass analysers is a powerful tool of increasing relevance and is often used for the precise analysis of protein mixtures ${ }^{44}$. To this end, we have recently developed a straightforward sheath flow CE-MS interface for separation with high-sensitivity Orbitrap mass detection ${ }^{45}$. Tandem MS measurements (MS/MS) were performed to verify the detected peptides and determine peptide sequences.

After identifying $\mathrm{SO}_{2}$ as an interesting alternative solvent for prebiotic peptide condensation and optimising the reaction conditions, we extended the system to the complete set of 20 proteinogenic amino acids. In this way, the formation of any peptide in question is possible, and thus a meaningful evaluation of the system is achievable. For a conclusive interpretation of the results, we compared the dipeptide mixtures formed with those of the corresponding reaction under equivalent conditions in aqueous solution. We observed the same positive effect of urea on peptide formation in aqueous solution, so it was added to the initial SIPF conditions. Thus, the reaction time, the initial amino acid and urea concentration, and the sample concentration during analysis were identical in both solvents. However, in a few cases the conditions differed based on previous findings: In the SIPF reaction, $\mathrm{CuCl}_{2}$ and $\mathrm{NaCl}$ cannot be used in stoichiometric amounts, as there are threshold concentrations that must be present for product formation to occur at all. As shown earlier, even minute amounts of $\mathrm{CuCl}_{2}$ induce peptide formation in $\mathrm{SO}_{2}$ and the hygroscopic environment is sufficient to make $\mathrm{NaCl}$ obsolete. Therefore, fixed $\mathrm{CuCl}_{2}$ and $\mathrm{NaCl}$ concentrations of $400 \mathrm{mM}$ and $4.4 \mathrm{M}$ were used in water, while stoichiometric amounts of $\mathrm{CuCl}_{2}$ were used in $\mathrm{SO}_{2}(1 / n$ equivalents for mixtures of $n$ amino acids). $\mathrm{NaCl}$ 
was not used at all. Furthermore, room temperature has been shown to be sufficient for a successful reaction in $\mathrm{SO}_{2}$, whereas a temperature of $85^{\circ} \mathrm{C}$ is used in water.

Thus, in addition to the complete set of 20 proteinogenic amino acids, four subgroups were analysed as a function of side-chain properties. The amino acids were divided into a nonpolar, polar-neutral, alkaline, and acidic group and analysed separately (Fig. 2). In addition, a selected mixture of amino acids that were probably first present on the early Earth ${ }^{46,47}$ was examined. The total concentration of amino acids and urea was maintained at $400 \mathrm{mM}$ in all cases and samples were taken after $7 \mathrm{~d}$ and $21 \mathrm{~d}$.

The reaction of the nonpolar mixture in $\mathrm{SO}_{2}$ yielded every possible dipeptide of the product spectrum (Fig. 3a). After $7 \mathrm{~d}$, few valine-containing dipeptides were not yet formed, but these appear after $21 \mathrm{~d}$. Similarly, most of the possible products are also formed in aqueous solution. However, tryptophan (W) shows low stability in solution and is susceptible to side reactions, especially in combination with metals or high temperatures ${ }^{48}$. After $7 \mathrm{~d}$, this amino acid was rarely detected and accordingly dipeptide formation of $\mathrm{W}$ in aqueous solution occurred only very rarely. Apart from this, traces of tripeptides were detected in both reaction media.

The polar-neutral mixture was less reactive in both reaction media (Fig. $3 \mathrm{~b}$ ). In $\mathrm{SO}_{2}$, tyrosine (Y) showed less reactivity, possibly due to its low solubility in the absence of acidic or basic conditions. The formation of dipeptides containing cysteine (C) could not be observed; however, its oxidation to cystine could be confirmed by MS/MS (for MS data, see Supplementary Fig. 38). Furthermore, cystine formed peptide bonds with other polar amino acids as both $\mathrm{N}$ - and C-terminal residues (Supplementary Fig. 39). Apart from this, all dipeptides were observed after $21 \mathrm{~d}$. In aqueous solution, no cysteines could be detected either, but no disulphide bridges were formed here. Furthermore, the hydrolysis of asparagine $(\mathrm{N})$ and glutamine $(\mathrm{Q})$ to the respective acidic amino acids aspartic acid (D) and glutamic acid (E) led to a rapid decrease in their concentration. This strongly inhibited dipeptide formation with $Q$ in particular. Small amounts of tripeptides could be detected in both reaction media, 
especially those containing two glycine molecules. Importantly, they were formed in higher amounts in $\mathrm{SO}_{2}$ so that their sequence determination was possible (GGG, GGN, NGG, GGQ, QGG, GGS, GSG, SGG, GGT, GTG and TGG).

For the alkaline and acidic mixtures, almost all possible dipeptides could be detected. The aqueousalkaline mixture showed a strong preference for the incorporation of lysine, as no other dipeptides were confirmed. No tripeptide formation was detected in either solvent (Supplementary Fig. 24-27).

In both media numerous dipeptides were formed from the prebiotic mixture containing the amino acids proline $(P)$, serine $(S)$, threonine $(T)$, leucine $(L)$, isoleucine $(I), A, D, E, G$ and $V$, which were predominant on the early Earth (Fig. 4). The acidic amino acids were less favoured for incorporation, especially in $\mathrm{SO}_{2}$. Instead, $\mathrm{G}, \mathrm{I}$ and $\mathrm{L}$ were the most reactive in both solvents, as well as $\mathrm{P}$ in $\mathrm{SO}_{2}$ and $\mathrm{V}$ in water. The preference of one sequence over the inverted sequence was observed for several peptides (e.g., VT > TV, DG > GD). The complete dipeptide product tables with differentiation of the peptide sequences can be found in the supplementary information (Fig. 19-36).

Finally, we performed the reaction with all 20 proteinogenic amino acids to investigate the reactivity of the overall system (Fig. 5). Some trends from the smaller subgroups remained in the overall mixture, while new observations were also made. In water, the lack of reactivity of $W, Q, C$ and $H$ was still noticeable. In contrast, $\mathrm{Y}$ and the acidic amino acids were rather inactive in $\mathrm{SO}_{2}$. Cysteine formed few dipeptides, but oxidation to cystine and further condensation with other amino acids was repeatedly observed. In both solvents, many dipeptides with amino acids I, L, G, V and K were detected. Other dipeptides were preferentially formed in one of the two environments (e.g., D was more reactive in water, while $\mathrm{P}$ and $\mathrm{H}$ were more reactive in $\mathrm{SO}_{2}$ ). The composition of the amino acid mixture also affected product formation. The high activity of $\mathrm{V}$ in the total mixture was not predictable from the observations we made in the non-polar mixture. $\mathrm{In} \mathrm{SO}_{2}, \mathrm{~V}$ was the least reactive amino acid, while $\mathrm{W}$ formed fewer peptides in the total mixture. In water, far fewer dipeptides of phenylalanine (F) could be detected in the total mixture than in the subgroup. Because the amino acid concentration in all 
213 reactions was always related to the total number of amino acids, the concentration of a single amino

214 acid in the total mixture was lower than in the other mixtures. Therefore, some dipeptides were

215 formed in the subgroups and not in the total mixture (e.g., MI, ST, HH, DD), and this effect was more pronounced in water than in $\mathrm{SO}_{2}$. To investigate this finding in more detail, we performed the reactions of the total mixture at a lower initial amino acid concentration $(50 \mathrm{mM}$ versus $400 \mathrm{mM}$ total concentration; $2.5 \mathrm{mM}$ versus $20 \mathrm{mM}$ per amino acid). The previous experiments already showed that high yields can be obtained in $\mathrm{SO}_{2}$ even at these low concentrations. However, it is only in comparison

220 with the reaction in aqueous solution that the full potential of $\mathrm{SO}_{2}$ becomes obvious (Fig. 5). In water, 221 only very limited peptide formation is possible, resulting in remarkably few dipeptides. In $\mathrm{SO}_{2}$, fewer peptides were detected, but nevertheless peptide formation was observed for almost all amino acids, which demonstrates the effective reaction conditions impressively. 


\section{Discussion}

The formation of functional oligomer chains from single monomer building blocks remains one of the fundamental challenges that must be pursued to understand the evolution of life. In this study, we have presented an alternative reaction medium to circumvent the often discussed thermodynamically inhibited peptide condensation in aqueous environments. On the early Earth, $\mathrm{SO}_{2}$ was released by volcanic emissions. In the Hadean eon, this was probably liquid, due to the higher atmospheric pressure. Therefore, $\mathrm{SO}_{2}$ is an attractive surrogate solvent for prebiotic chemistry. In our study, we were able to demonstrate effective peptide coupling by metal catalysis up to tetrapeptides starting from single amino acids.

The diverse functions that a peptide can fulfil are determined solely by its sequence. The use of many different amino acids enables the formation of an even greater variety of peptides and peptide sequences. To our knowledge, the simultaneous conversion and detailed analysis of the complete set of all proteinogenic amino acids under prebiotic conditions has not been studied before. The results of this study show that the reactivity of amino acids cannot be considered in isolation. The investigation of complex mixtures reveals effects that would have been overlooked in reactions of single amino acids. In both environments studied, it was shown that the reactivity of the individual amino acid can depend on the mixture in which it is used. In $\mathrm{SO}_{2}$, valine was the least reactive amino acid in the nonpolar mixture. In the total mixture, however, it showed increased reactivity compared to $W$. Furthermore, it became clear that $\mathrm{V}$ showed exceptionally good overall reactivity with the other amino acids.

Furthermore, a detailed comparison of the two environments investigated could be made by applying complex mixtures of amino acids. The comparison showed that the different amino acid activities in the two solvents lead to different product distributions. Factors influencing the amino acid reactivity in both media are the stability as well as the solubility of the reagents $(Y, W)$, side reactions such as hydrolysis $(Q, N)$, the redox reaction of cysteine and the nucleo-/electrophilicity of the amino acids. 
The addition of urea led to increased dipeptide detection in both solvents. The formation of cysteines and cystine could only be observed in $\mathrm{SO}_{2}$. Interestingly, after oxidation to cystine, a further reaction to cystine tripeptides could be confirmed, providing sulphur bridges and the possibility for additional structural elements. Noteworthy is the increased formation of proline peptides, which are known for their catalytic activity ${ }^{49}$. Most importantly, each of the 20 amino acids could be incorporated into $\mathrm{SO}_{2}$, which could not be shown for the SIPF reaction. In general, the reaction conditions in $\mathrm{SO}_{2}$ led to a higher reactivity of the amino acids and a greater variety of products. However, the superiority of the solvent is not only reflected in the increased reactivity of the reactants but is particularly evident when compared to the corresponding reaction conditions in water, which differ in several crucial aspects. Most striking is the efficiency of peptide formation over a wide range of initial reactant concentrations. Comparatively low initial reactant concentrations of $50 \mathrm{mM}$ resulted in $2.9 \%$ dialanine yield and reducing the amino acid concentration in the total mixture to even $2.5 \mathrm{mM}$ still resulted in extensive dipeptide formation. The metal salt could be used in catalytic amounts and other additives like exploitative conversion at room temperature. All these findings increase the plausibility of the solvent in a prebiotic context, which is further supported by the successful product formation using the Hadean mineral covellite instead of $\mathrm{CuCl}_{2}$. Accordingly, fewer reactants in minor concentrations at a lower temperature are required for efficient peptide condensation in $\mathrm{SO}_{2}$. The effectiveness of the reaction under simple conditions suggests a high potential for peptide formation in a volcanic $\mathrm{SO}_{2}$ environment.

\section{Methods}

\subsection{Amino acid mixtures}

273 For the reactions containing mixtures of amino acids, equimolar amounts of amino acids were

274 homogenised by ball milling in a $20 \mathrm{~mL}$ stainless steel jar equipped with ten stainless steel balls 
(diameter $=10 \mathrm{~mm}$ ) at $400 \mathrm{rpm}$ for $10 \mathrm{~min}$ in the planetary ball mill Pulverisette 7 premium line (Fritsch $\mathrm{GmbH}$, Idar-Oberstein, Germany). The detailed list of sample weights can be found in the Supplementary Information.

\subsection{Peptide condensation reactions}

Peptide formation in aqueous solution was carried out in $4 \mathrm{~mL}$ glass vials with magnetic stir bar. To the respective amino acid mixture $(1.20 \mathrm{mmol}$ in total, $1.00 \mathrm{eq})$ urea $(72.1 \mathrm{mg}, 1.20 \mathrm{mmol}, 1.00 \mathrm{eq})$ and aqueous stock solutions of $\mathrm{NaCl}(2.64 \mathrm{~mL}, 5.00 \mathrm{M})$ and $\mathrm{CuCl}_{2}(360 \mu \mathrm{L}, 3.33 \mathrm{M})$ were added. The vials were sealed and placed in a metal block at $85^{\circ} \mathrm{C}$. The reactions were stirred for $21 \mathrm{~d}$ and samples were taken after 7 and $21 \mathrm{~d}$. All experiments were performed three times.

Reactions in $\mathrm{SO}_{2}$ (N38, 99.98\%, Air Liquide Germany) were performed in stainless steel pressure apparatuses (Supplementary Fig. 1). A $3.5 \mathrm{~mL}$ test tube equipped with a magnetic stir bar was filled with glycine (90.1 mg, $1.20 \mathrm{mmol}, 1.00$ eq.), L-alanine (106.9 mg, $1.20 \mathrm{mmol}, 1.00$ eq.), urea (72.1 mg, $1.20 \mathrm{mmol}, 1.00$ eq.) and $\mathrm{CuCl}_{2}(161.3 \mathrm{mg}, 1.20 \mathrm{mmol}, 1.00$ eq.) or optionally with covellite (114.7 mg, $20.9 \mathrm{mmol} / \mathrm{g}$ ). For reactions with complex mixtures, the particular amino acid mixture consisting of $n$ amino acids ( $1.20 \mathrm{mmol}$ in total, 1.00 eq.), urea ( $72.1 \mathrm{mg}, 1.20 \mathrm{mmol}, 1.00$ eq.) and $\mathrm{CuCl}_{2}$ (1/n eq.) were added to the test tube instead. The test tube was inserted in the pressure apparatus, evacuated and refilled with nitrogen three times. At $-76^{\circ} \mathrm{C} 3 \mathrm{~mL} \mathrm{SO}$, were condensed into the reaction chamber. The valves were closed, and the reaction carried out at room temperature under stirring for 1 to $21 \mathrm{~d}$. Subsequently, the $\mathrm{SO}_{2}$ was condensed into the storage chamber and the remaining solid dried in vacuo. The solvent was reused up to five times for further reactions. The test tube was removed from the apparatus and the product mixture stored at $-18^{\circ} \mathrm{C}$ until analysis.

\subsection{CE-MS/MS analysis}

Before measurement, the samples were diluted according to the initial amino acid concentration. In all cases, the concentration of each amino acid of the mixture in the sample vial was $1 \mathrm{mM}$. The product mixtures were analysed using an Agilent 7100 CE system coupled to a Thermo Scientific Q Exactive Plus 
mass spectrometer with a custom-made sheath-flow interface, which was described in a previous study ${ }^{45}$. The electrophoretic separations were performed in positive polarity mode at $25^{\circ} \mathrm{C}$ with an aqueous acetic acid solution (2 M) as background electrolyte (BGE). To avoid peptide adsorption linear polyacrylamide-coated capillaries with a total length of $80 \mathrm{~cm}$ were used whose preparation was described elsewhere ${ }^{50}$. Before their first utilization, a short piece of the outer polyimide coating was removed at the MS end of the capillaries and they were conditioned with deionised water (2 min), aqueous $\mathrm{H}_{3} \mathrm{PO}_{4}(10 \mathrm{mM}, 5 \mathrm{~min})$, deionised water (2 min) and BGE (2 min). Between measurements the capillaries were flushed again with aqueous $\mathrm{H}_{3} \mathrm{PO}_{4}(10 \mathrm{mM}, 30 \mathrm{~s})$, deionised water (1 min) and $\mathrm{BGE}$ ( $2 \mathrm{~min}$ ). Sample injection was performed by applying 30 mbar pressure for $10 \mathrm{~s}$. To separate the peptide mixture, a voltage of $30 \mathrm{kV}$ and a constant assisting pressure of $30 \mathrm{mbar}$ was applied to the $\mathrm{CE}$ inlet. For establishing a stable electrospray an external voltage of $3.2 \mathrm{kV}$ was applied to the stainlesssteel emitter while the sheath liquid was delivered at $3 \mu \mathrm{L} / \mathrm{min}$. The sheath liquid consisted of deionised water and isopropanol (50:50) with 0.05\% formic acid.

Mass spectra were recorded in positive mode with a resolution of 70000 in the mass range $\mathrm{m} / \mathrm{z} 122$ 750. The temperature of the ion transfer capillary was set to $140^{\circ} \mathrm{C}$ while a minimal flow of sweep gas was applied. The S-lens RF level was adjusted to 50. For dipeptide fragmentation data dependant MS/MS measurements with inclusion lists containing all possible peptide products were performed. The MS/MS spectra were measured with a resolution of 17500 using a normalized collision energy of 30\%. The mass spectra were evaluated using Thermo Xcalibur software 4.1.

\subsection{CE analysis}

Dipeptide quantification of the alanine-glycine system was performed using the Agilent $7100 \mathrm{CE}$ system. Before measurement, samples were diluted to an amino acid concentration of $10 \mathrm{mM}$. The analysis was conducted in positive polarity mode at $25^{\circ} \mathrm{C}$ using a conductivity detector. Separations were accomplished on bare fused silica capillaries with a total length of $80 \mathrm{~cm}$ and aqueous acetic acid (2 M) as BGE by applying a voltage of $30 \mathrm{kV}$. Samples were injected by applying $30 \mathrm{mbar}$ for $10 \mathrm{~s}$. 
325 Calibration curves of $G G, A G+G A$ and $A A$ were recorded in triplicates and 4-hydroxyproline $(100 \mu M)$

326 was used as internal standard. New capillaries were conditioned with deionised water, aqueous $\mathrm{NaOH}$

327 (0.1 M), deionised water and BGE (each $5 \mathrm{~min}$ ). Between measurements, capillaries were flushed with

328 aqueous $\mathrm{NaOH}(0.1 \mathrm{M})$, deionised water and $\mathrm{BGE}$ (each $2 \mathrm{~min}$ ). CE electropherograms were evaluated 329 using CEval $0.6 \mathrm{~g}^{51}$ and OriginPro 2018G. 
1 Frenkel-Pinter, M., Samanta, M., Ashkenasy, G. \& Leman, L. J. Prebiotic Peptides: Molecular Hubs in the Origin of Life. Chem. Rev. 120, 4707-4765 (2020).

2 Miller, S. L. A Production of Amino Acids Under Possible Primitive Earth Conditions. Science 117, 528-529 (1953).

3 Parker, E. T. et al. Primordial synthesis of amines and amino acids in a 1958 Miller $\mathrm{H}_{2} \mathrm{~S}$-rich spark discharge experiment. Proc. Natl. Acad. Sci. 108, 5526-5531 (2011).

4 Harada, K. \& Fox, S. W. Thermal Synthesis of Natural Amino-Acids from a Postulated Primitive Terrestrial Atmosphere. Nature 201, 335-336 (1964).

5 Zhang, X. et al. Prebiotic Synthesis of Glycine from Ethanolamine in Simulated Archean Alkaline Hydrothermal Vents. Orig. Life Evol. Biosph. 47, 413-425 (2017).

6 Martins, Z., Modica, P., Zanda, B. \& d'Hendecourt, L. L. S. The amino acid and hydrocarbon contents of the Paris meteorite: Insights into the most primitive CM chondrite. Meteorit. Planet. Sci. 50, 926-943 (2015).

7 Cronin, J. R. \& Pizzarello, S. Enantiomeric Excesses in Meteoritic Amino Acids. Science 275, 951955 (1997).

8 Glavin, D. P. \& Dworkin, J. P. Enrichment of the amino acid L-isovaline by aqueous alteration on Cl and CM meteorite parent bodies. Proc. Natl. Acad. Sci. 106, 5487-5492 (2009).

9 Pizzarello, S., Schrader, D. L., Monroe, A. A. \& Lauretta, D. S. Large enantiomeric excesses in primitive meteorites and the diverse effects of water in cosmochemical evolution. Proc. Natl. Acad. Sci. 109, 11949-11954 (2012).

10 Fox, S. W. \& Harada, K. Thermal Copolymerization of Amino Acids to a Product Resembling Protein. Science 128, 1214-1214 (1958). 
11 Rodriguez-Garcia, M. et al. Formation of oligopeptides in high yield under simple programmable conditions. Nat. Commun. 6, 8385-8391 (2015).

12 Ferris, J. P., Hill, A. R., Liu, R. \& Orgel, L. E. Synthesis of long prebiotic oligomers on mineral surfaces. Nature 381, 59-61 (1996).

13 Lambert, J.-F. Adsorption and Polymerization of Amino Acids on Mineral Surfaces: A Review. Orig. Life Evol. Biosph. 38, 211-242 (2008).

14 Frenkel-Pinter, M. et al. Selective incorporation of proteinaceous over nonproteinaceous cationic amino acids in model prebiotic oligomerization reactions. Proc. Natl. Acad. Sci., 116, 16338-16346 (2019).

15 Canavelli, P., Islam, S. \& Powner, M. W. Peptide ligation by chemoselective aminonitrile coupling in water. Nature 571, 546-549 (2019).

16 Brack, A. Selective emergence and survival of early polypeptides in water. Orig. Life Evol. Biosph. 17, 367-379 (1987).

17 Stolar, T. et al. Mechanochemical prebiotic peptide bond formation. Angew. Chem. Int. Ed. 60, $12727-12731$ (2021).

18 Schwendinger, M. G. \& Rode, B. M. Possible Role of Copper and Sodium Chloride in Prebiotic Evolution of Peptides. Anal. Sci. 5, 411-414 (1989).

19 Jakschitz, T. A. E. \& Rode, B. M. Chemical evolution from simple inorganic compounds to chiral peptides. Chem. Soc. Rev. 41, 5484-5489 (2012).

20 Ochiai, E.-I. The evolution of the environment and its influence on the evolution of life. Orig. Life 9, 81-91 (1978).

21 Hazen, R. M. Paleomineralogy of the Hadean Eon: A preliminary species list. Am. J. Sci. 313, 807843 (2013). 
22 Yamagata, Y., Watanabe, H., Saitoh, M. \& Namba, T. Volcanic production of polyphosphates and its relevance to prebiotic evolution. Nature 352, 516-519 (1991).

23 Šponer, J. E. et al. Emergence of the First Catalytic Oligonucleotides in a Formamide-Based Origin Scenario. Chem. Eur. J. 22, 3572-3586 (2016).

24 Saladino, R., Crestini, C., Pino, S., Costanzo, G. \& Di Mauro, E. Formamide and the origin of life. Phys. Life Rev. 9, 84-104 (2012).

25 Menor-Salván, C. \& Marín-Yaseli, M. R. Prebiotic chemistry in eutectic solutions at the water-ice matrix. Chem. Soc. Rev. 41, 5404-5415 (2012).

26 Gull, M., Zhou, M., Fernández, F. M. \& Pasek, M. A. Prebiotic Phosphate Ester Syntheses in a Deep Eutectic Solvent. J. Mol. Evol. 78, 109-117 (2014).

27 Kasting, J. F., Zahnle, K. J., Pinto, J. P. \& Young, A. T. Sulfur, ultraviolet radiation, and the early evolution of life. Orig. Life Evol. Biosph. 19, 95-108 (1989).

28 Walker, J. C. G. \& Brimblecombe, P. Iron and sulfur in the pre-biologic Ocean. Precambrian Res. 28, 205-222 (1985).

29 Marcq, E., Bertaux, J.-L., Montmessin, F. \& Belyaev, D. Variations of sulphur dioxide at the cloud top of Venus's dynamic atmosphere. Nat. Geosci. 6, 25-28 (2013).

30 Bergstrom, F. W. The Boiling Points of Ammonia, Sulfur Dioxide and Nitrous Oxide. J. Phys. Chem. 26, 876-894 (1922).

31 Bergstrom, F. W. The Vapor Pressure of Sulfur Dioxide and Ammonia. J. Phys. Chem. 26, 358-376 (1922).

32 Sleep, N. H. The Hadean-Archaean Environment. Cold Spring Harb. Perspect. Biol. 2, a002527 (2010). 
33 Kawai, D., McLendon, D. C., Kim, H.-J. \& Benner, S.A., Hydroxymethanesulfonate from Volcanic Sulfur Dioxide: A "Mineral" Reservoir for Formaldehyde and Other Simple Carbohydrates in Prebiotic Chemistry. Astrobiology 19, 506-516 (2019).

34 Chen, F. \& Yang, D. Condensation of amino acids to form peptides in aqueous solution induced by the oxidation of sulfur(iv): an oxidative model for prebiotic peptide formation. Orig. Life Evol. Biosph. 37, 47-54 (2007).

35 Ranjan, S., Todd, Z. R., Sutherland, J. D. \& Sasselov, D. D. Sulfidic Anion Concentrations on Early Earth for Surficial Origins-of-Life Chemistry. Astrobiology 18, 1023-1040 (2018).

36 Nanda, J. et al. Emergence of native peptide sequences in prebiotic replication networks. Nat. Commun. 8, 434 (2017).

37 Mayr, H., Gorath, G. \& Bauer, B. Liquid Sulfur Dioxide as a Lewis-Acidic Solvent for the Alkylation and Alkoxyalkylation of Allylsilanes. Angew. Chem. Int. Ed. 33, 788-789 (1994).

38 Danger, G., Plasson, R. \& Pascal, R. Pathways for the formation and evolution of peptides in prebiotic environments. Chem. Soc. Rev. 41, 5416-5429 (2012).

39 Lohrmann, R. Formation of urea and guanidine by irradiation of ammonium cyanide. J. Mol. Evol. 1, 263-269 (1972).

40 Lowe, C. U., Rees, M. W. \& Markham, R. Synthesis of Complex Organic Compounds from Simple Precursors: Formation of Amino-Acids, Amino-Acid Polymers, Fatty Acids and Purines from Ammonium Cyanide. Nature 199, 219-222 (1963).

41 Gull, M., Omran, A., Feng, T. \& Pasek, M. A. Silicate-, Magnesium Ion-, and Urea-Induced Prebiotic Phosphorylation of Uridine via Pyrophosphate; Revisiting the Hot Drying Water Pool Scenario. Life $10,122(2020)$.

42 Lohrmann, R. \& Orgel, L. E. Urea-Inorganic Phosphate Mixtures as Prebiotic Phosphorylating Agents. Science 171, 490-494 (1971). 
43 Hua, L., Zhou, R., Thirumalai, D. \& Berne, B. J. Urea denaturation by stronger dispersion interactions with proteins than water implies a 2-stage unfolding. Proc. Natl. Acad. Sci. 105, 16928-16933 (2008).

44 Zhang, Z., Qu, Y. \& Dovichi, N. J. Capillary zone electrophoresis-mass spectrometry for bottom-up proteomics. Trends Analyt. Chem. 108, 23-37 (2018).

45 Sauer, F., Sydow, C. \& Trapp, O. A robust sheath-flow CE-MS interface for hyphenation with Orbitrap MS. Electrophoresis 41, 1280-1286 (2020).

46 Higgs, P. G. \& Pudritz, R. E. A Thermodynamic Basis for Prebiotic Amino Acid Synthesis and the Nature of the First Genetic Code. Astrobiology 9, 483-490 (2009).

47 Trifonov, E. N. The origin of the genetic code and of the earliest oligopeptides. Res. Microbiol. 160, 481-486 (2009).

48 Bellmaine, S., Schnellbaecher, A. \& Zimmer, A. Reactivity and degradation products of tryptophan in solution and proteins. Free Radic. Biol. Med. 160, 696-718 (2020).

49 Schnitzer, T., Wiesner, M., Krattiger, P., Revell, J. D. \& Wennemers, H. Is more better? A comparison of tri- and tetrapeptidic catalysts. Org. Biomol. Chem. 15, 5877-5881 (2017).

50 Zhu, G., Sun, L. \& Dovichi, N. J. Thermally-initiated free radical polymerization for reproducible production of stable linear polyacrylamide coated capillaries, and their application to proteomic analysis using capillary zone electrophoresis-mass spectrometry. Talanta 146, 839-843 (2016).

51 Dubský, P., Ördögová, M., Malý, M. \& Riesová, M. CEval: All-in-one software for data processing and statistical evaluations in affinity capillary electrophoresis. J. Chromatogr. A 1445, 158-165 (2016). 


\section{Acknowledgements}

We acknowledge financial support from the Ludwig-Maximilians-University Munich, the Max-PlanckSociety (Max-Planck-Fellow Research Group Origins of Life), the Volkswagen Stiftung (Initiating Molecular Life), the Deutsche Forschungsgemeinschaft DFG/German Research Foundation (Project-ID 364653263 - TRR 235, Emergence of Life) and Germany's Excellence Strategy (ORIGINS, EXC-2094 390783311).

\section{Author Contributions}

O.T. conceived the idea and directed the project. O.T., F.S. and M.H. designed the experiments. F.S. and C.A.L. performed the experiments in aqueous salt solution. M.H. and C.S. carried out the reactions in $\mathrm{SO}_{2}$. F.S. and M.H. developed and performed the analytical separation and identification methods. C.S., F.S. and A.F.S. validated and performed the quantification. F.S., M.H., C.S. and A.F.S. evaluated the data. M.H., F.S. and O.T. wrote the manuscript. All authors discussed the results and edited the manuscript.

\section{Competing Interests}

The authors declare no conflict of interest.

\section{Additional information}

Supplementary Information is available for this paper. Correspondence and requests for materials should be addressed to Oliver Trapp. 


\section{Figures}

Figure 1. Dipeptide yields in $\mathrm{SO}_{2}$. a Peptide yields in dependence of the reaction time are shown with initial $\mathrm{A}, \mathrm{G}$ and $\mathrm{CuCl}_{2}$ concentrations of $400 \mathrm{mM}$. b Peptide yields in dependence of the initial amino acid and $\mathrm{CuCl}_{2}$ concentrations after $7 \mathrm{~d}$ are displayed. Error bars are \pm s.d. after two measurements.

a

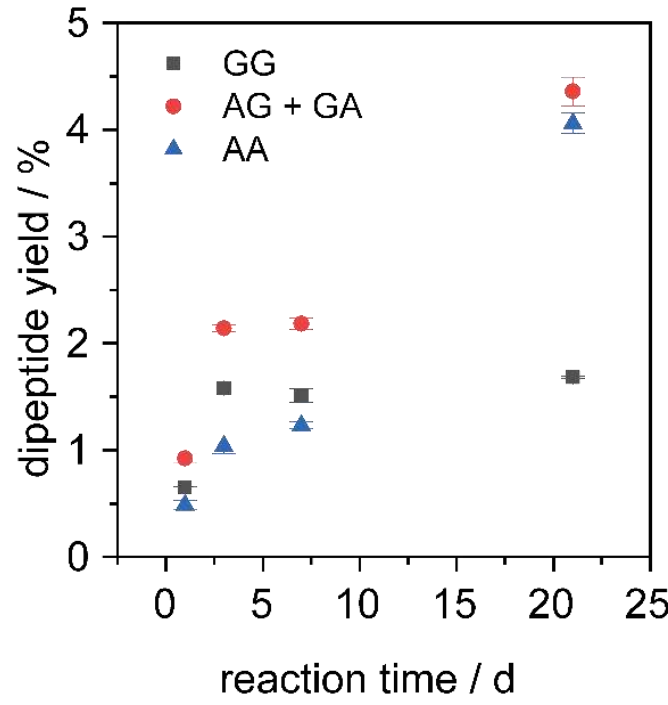

b

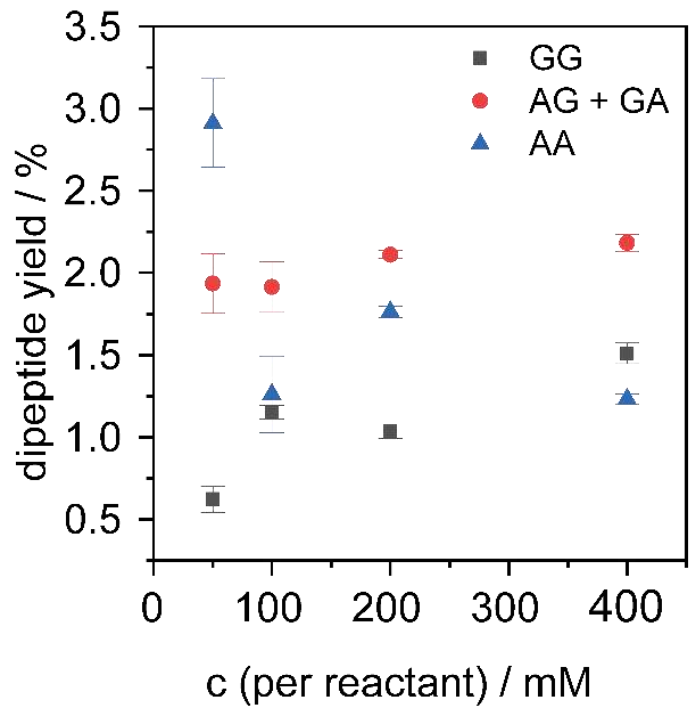


Figure 2. Experimental and analytical routine. The proteinogenic amino acids were divided into subgroups based on their properties. Each of these complex subgroups was reacted under the same

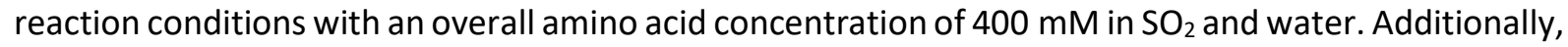
the complete set of the proteinogenic amino acids was used in two different overall amino acid concentrations $(400 \mathrm{mM}$ and $50 \mathrm{mM})$. After electrophoretic separation of the reaction mixtures after 7 and $21 \mathrm{~d}$, the resulting dipeptides were confirmed by tandem mass spectrometry allowing for a thorough comparison of the product distribution.
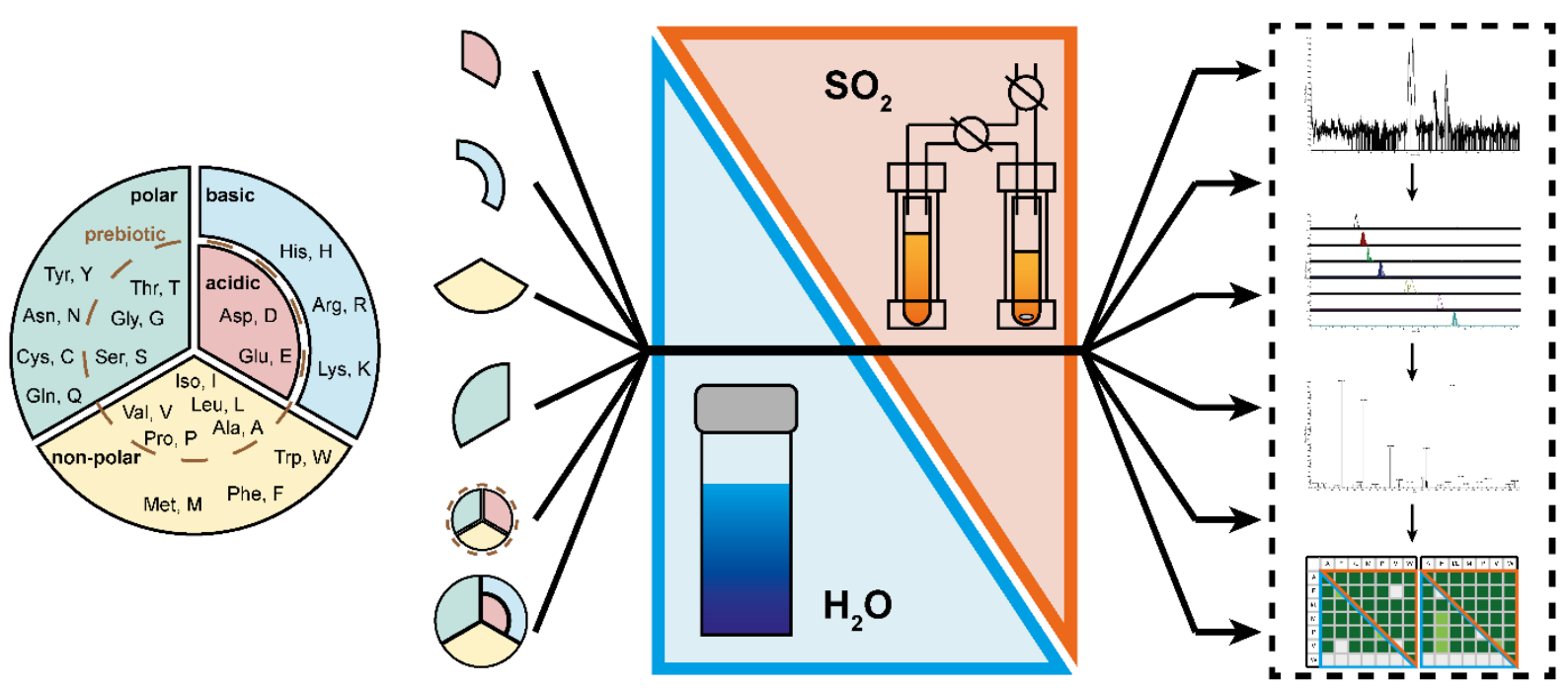
Figure 3. Product spectrum of the non-polar and polar-neutral amino acid mixture. Comparison of the detected dipeptides of copper-catalysed peptide condensation in $\mathrm{SO}_{2}$ and water after 7 and $21 \mathrm{~d}$ starting from the non-polar (a) or polar neutral (b) mixture $(\square=$ confirmed by MS/MS, $\square=$ traces, $\square$ = not detected, $=$ cystine-peptides confirmed by MS/MS, $\mathbb{Z}=$ cystine-peptide traces). Amino acids are represented corresponding to the one letter code.

a

\section{C-terminus}

$7 \mathrm{~d}$

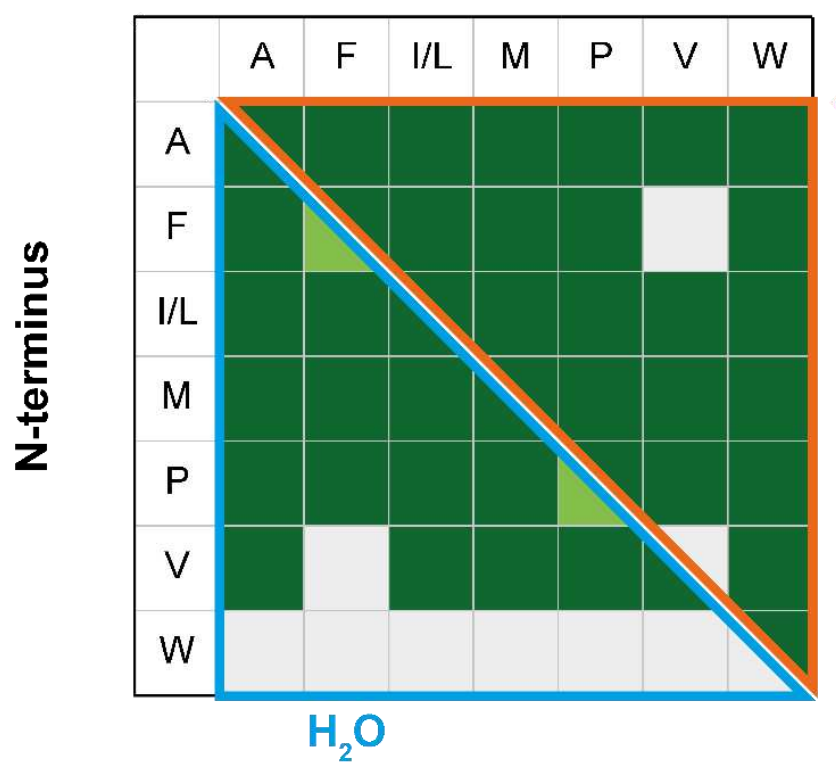

$21 \mathrm{~d}$

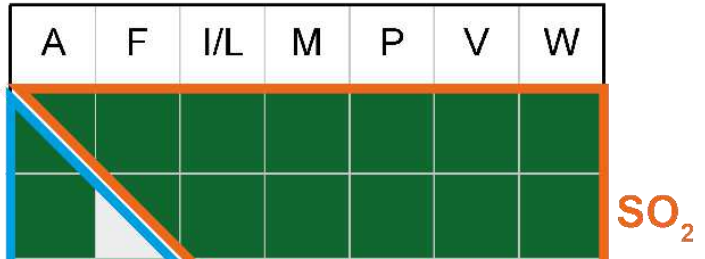

C-terminus

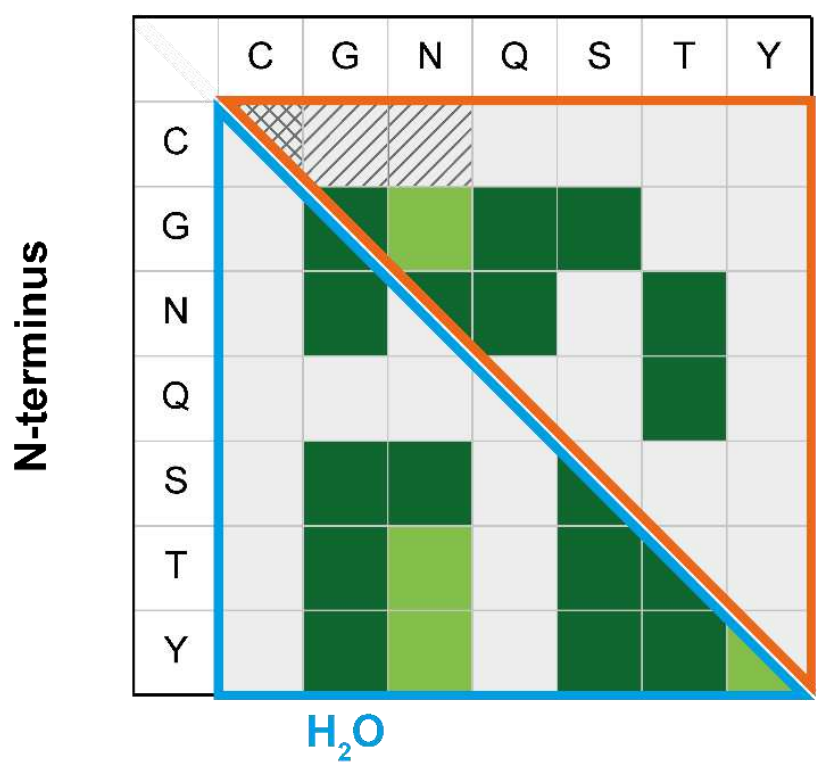

$21 \mathrm{~d}$

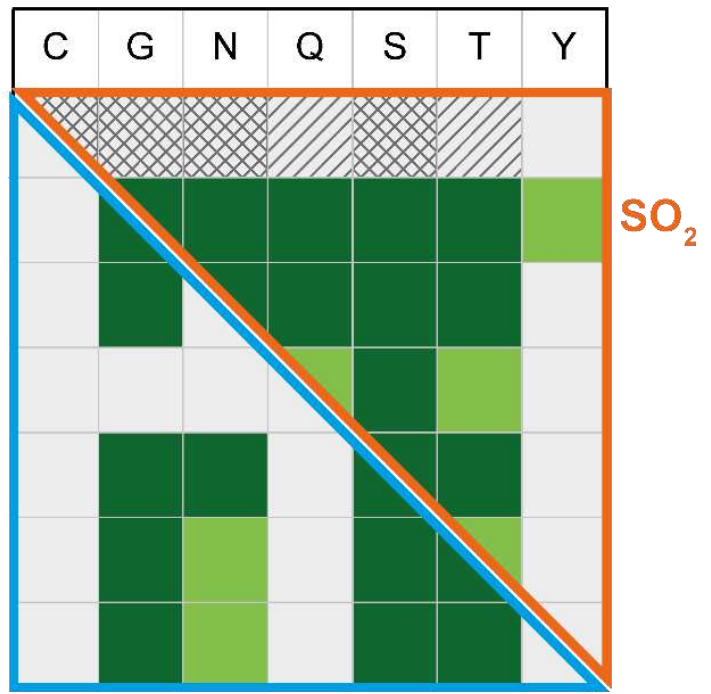


Figure 4. Product spectrum of the prebiotic amino acid mixture. Comparison of the detected dipeptides of copper-catalysed peptide condensation in $\mathrm{SO}_{2}$ and water after 7 and $21 \mathrm{~d}$ starting from the prebiotic amino acid mixture ( $\square=$ confirmed by MS/MS, $\square=$ traces, $\square=$ not detected). Amino acids are represented corresponding to the one letter code.

\section{C-terminus}

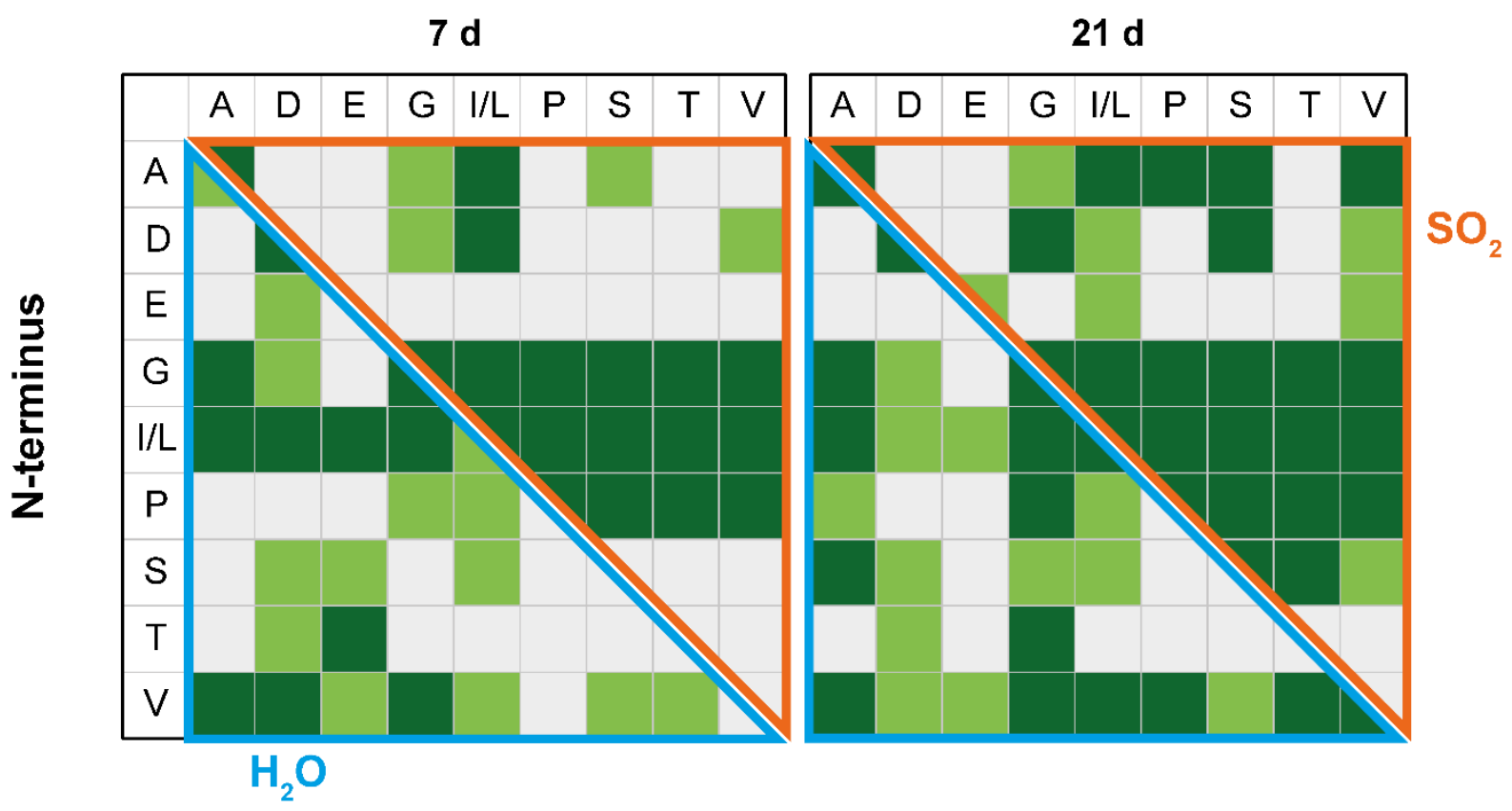


Figure 5. Product spectrum of the complete amino acid mixture. Comparison of the detected dipeptides of copper-catalysed peptide condensation in $\mathrm{SO}_{2}$ and water after $21 \mathrm{~d}$ starting from $400 \mathrm{mM}$ or $50 \mathrm{mM}$ of the complete set of proteinogenic amino acids $(\square=$ confirmed by MS/MS, $=$ traces, $\square=$ not detected, $\mathbb{Z} \angle$ = cystine-peptide traces). The $\mathrm{CuCl}_{2}$ concentration in $\mathrm{SO}_{2}$ was $5 \mathrm{~mol} \%$ in both experiments. Amino acids are represented corresponding to the one letter code.

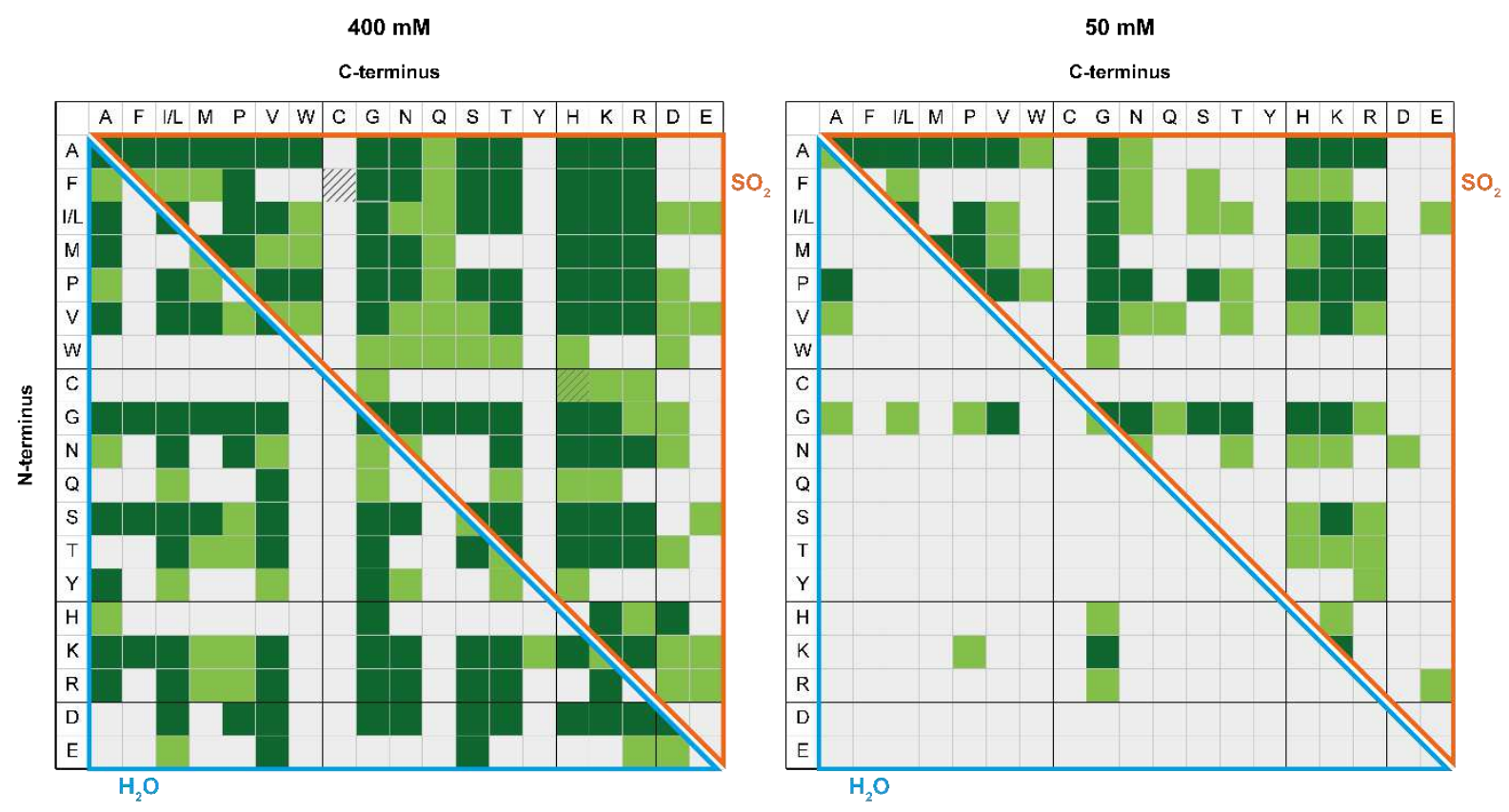




\section{Supplementary Files}

This is a list of supplementary files associated with this preprint. Click to download.

- SIPeptidesV9.pdf 